\title{
Innovación para la integración a las redes de proveeduría de las empresas multinacionales
}

\section{Innovation for integration into supplier networks of multinational companies}

\author{
Jaime Olea Miranda*, Alejandro Valenzuela Valenzuela, \\ María de los Ángeles Navarrete Hinojosa
}

Universidad de Sonora, México

Recibido el 19 de enero de 2016; aceptado el 14 de marzo de 2016

Disponible en Internet el 11 de diciembre de 2017

\section{Resumen:}

El propósito del presente trabajo es demostrar que la innovación promueve la probabilidad de que las pequeñas empresas formen parte de las redes de proveeduría de las grandes empresas asentadas en los parques industriales locales. En particular, se demuestra a través del diseño de un modelo de regresión logística que a mayor número de tipos de innovaciones (de producto, proceso, administración y comercialización), mayor es la probabilidad de que una pequeña empresa cualquiera elegida al azar sea proveedora de las empresas multinacionales que encabezan redes globales de valor. Las pruebas de hipótesis se realizan sobre la base de una muestra aleatoria de pequeñas empresas metalmecánicas y de tecnologías de la información de Sonora, basados en los resultados de investigaciones anteriores, donde se demuestra que este tipo de empresas están en mejores condiciones de participar en las redes de proveeduría de las grandes empresas.

Palabras clave: Innovación, Cadenas Globales de Valor, Pymes, empresas multinacionales. Códigos JEL: O14, O31, C25

\footnotetext{
*Autor para correspondencia.

Correo electrónico: jolea@industrial.uson.mx (J. Olea Miranda).

La revisión por pares es responsabilidad de la Universidad Nacional Autónoma de México.
} 


\begin{abstract}
Abstrac:
The main objective of this study is to demonstrate that innovation promotes the likelihood that small firms form part of the supplier networks of large companies based in local industrial parks. In particular, it is demonstrated through the design of a logistic regression model that more types of innovations (product, process, management and marketing) increase the probability that any randomly chosen small firms to be supplier of multinational companies leading global value chains. Hypothesis tests are performed on the basis of a random sample of small metalmecanic and of information technologies firms from Sonora, given that (as is showed by previous research) these firms are better able to participate in networks mentioned.
\end{abstract}

Keywords : Innovation, global value chains , SMEs, multinationals.

JEL Classification: O14, O31, C25

"No hay ciencia sin una distinción clara y operativa
entre variables independientes y variable dependiente".
Jon Elster (2011).
El desinterés. Tratado crítico del hombre económico.
México, Siglo XXI

\title{
Introducción
}

La participación de las pequeñas y medianas empresas (Pymes) en las redes de proveeduría de las trasnacionales (ETN) ubicadas en los mercados locales, es un tema de gran interés porque dinamiza y fortalece la economía regional. En particular, promueve el escalamiento de las pequeñas empresas por medio de mejoras en competitividad e incrementos en productividad (Bueno, 2012).

Ese escalamiento tiene lugar a través de muchos procesos, pero el elemento esencial es la innovación. Mucho se ha escrito al respecto, pero en este trabajo se busca explicar en el ámbito específico de la economía de Sonora, que la relación entre las Pymes metalmecánicas (MM) y de tecnologías de información (TI) y las redes globales de valor se fortalece y potencializa si las Pymes proveedoras de las ETN generan distintos tipos de innovaciones. Dicho de otra manera, si una empresa solo produce innovaciones de un tipo, digamos, de producto, sus probabilidades de pertenecer a las redes de proveeduría de las ETN son menores a las de aquellas que tienen diversificaciones en innovaciones de dos o más tipos (v.g. de producto, de proceso, de administración y de comercialización).

Es cierto que la innovación en los parques industriales locales se debe en gran medida a la derrama de conocimientos que realizan las grandes ETN (otros factores, como el espíritu emprendedor de los empresarios locales, las políticas públicas aplicadas y la existencia de una fuerza de trabajo calificada, también cuentan), pero la capacidad de absorción de conocimientos de las pequeñas empresas y su capacidad para convertir el conocimiento explícito en implícito y luego transformarlo en innovaciones es lo que les permite promoverse como candidatas a las redes de proveeduría de aquellas. 
El estudio se realizó con base en la información recabada en 68 pequeñas y medianas empresas (Pymes), de la rama Metalmecánica (MM) y Tecnologías de Información (TI) de Sonora a principios del 2015. Se realizó un muestreo aleatorio basado en un padrón depurado de empresas, como se explica en la parte metodológica, y las conclusiones podrían generalizarse a experiencias similares.

El documento se organiza en seis secciones, siendo esta introducción la primera. En el segundo apartado se hace un resumen del debate que se ha sostenido en torno al papel que desempeñan las pequeñas empresas en la economía. En el tercero se desarrolla con precisión el sector de las Pymes en Sonora, especialmente los sectores MM y TI. En el cuarto apartado se presenta la metodología, el diseño de la investigación, el enfoque teórico que sustenta la investigación y se presentan los modelos utilizados para las pruebas de hipótesis.

Por último, este artículo deriva de los resultados del proyecto de investigación "Transferencia de Conocimiento y Aprendizaje Tecnológico de las Pequeñas y Medianas Empresas (Pymes) locales en el entorno de las Redes Globales de Producción", financiado por Promep con registro 151329 .

\section{El debate sobre el papel de las PYMES}

El debate sobre el papel de las pequeñas empresas en la economía parece estar ya superado por los hechos. Más de 200 años después de que Marx escribiera el Manifiesto Comunista, los pequeños negocios no sólo no han desaparecido absorbidos por la concentración del capital, sino que se han revelado como elementos indispensable para el funcionamiento de un sistema regido por estructuras de mercado de competencia imperfecta (especialmente oligopólica y de competencia monopolística) (Matsuyama, 1995), donde las grandes empresas multinacionales juegan un papel de liderazgo de las redes globales de valor fijando las reglas de funcionamiento en mercados altamente globalizados. No es que carezcan de problemas, muy al contrario, como se verá más adelante, pero las Pymes han venido a constituir el intersticio que mantiene funcionando a las grandes empresas como parte del sistema económico.

El desempeño de esas empresas (su participación en el empleo, en el producto nacional, en la estabilidad de los mercados) apoyan las tesis del llamado enfoque dinámico que sostiene que la mayoría de las Pymes juegan un papel crucial en el funcionamiento de los mercados (Acs y Audretsch, 1993; Berger y Piore, 1980).

Este enfoque reconoce al menos dos funciones de las pequeñas empresas: primera, producir (y así lo hacen la mayoría de ellas) bienes intermedios para satisfacer la demanda (bajo el esquema de subcontratación) de las grandes corporaciones y, segunda, la introducción en la economía de productos avanzados (OCDE, 2010) dando impulso a la innovación en los sistemas regionales y nacionales (Armenteros, Medina, Ballesteros y Molina, 2012). Esta segunda función la logran generando productos y procesos nuevos o mejorados, a veces incluso radicales, que van empujando la economía hacia la frontera tecnológica.

Quienes sostienen el enfoque dinámico argumentan que esas pequeñas organizaciones productivas (y el espíritu empresarial), han jugado un rol en la economía mucho más importante de lo que se creía. Según esta aproximación, son cuatro las contribuciones específicas de ese tipo de negocios a los mercados industriales. Primera, el rol que juegan en el proceso de cambio tecnológico. Nelson y Winter (1982) argumentan que los pequeños 
negocios industriales hacen una significativa contribución empresarial en el sentido de que son la fuente de una considerable actividad innovadora. Segunda, la turbulencia que generan en los mercados, que no sólo crea una dimensión adicional a la competencia no captada por la visión estática de la estructura de mercados, sino que también genera mecanismos de regeneración sirviendo como agentes del cambio (Beesley y Hamilton, 1984) ${ }^{1}$. Tercera, relacionada con la segunda, es la promoción de la competitividad internacional a través de la creación de nuevos nichos de mercado (Brock and Evans, 1989). La cuarta contribución es su preponderante participación en los empleos de nueva creación (Storey, 1994) ${ }^{2}$. Se debe agregar a la lista anterior que las pequeñas empresas son la base de las redes de subcontratación en que se sustentan las corporaciones globales en los mercados locales.

\section{Los sectores metalmecánico y de tecnologías de la información}

Las pequeñas empresas han sido profusamente estudiadas. Se ha buscado entender su naturaleza (Brok y Evans, 1989; Coase, 193733; Storey, 1994), se ha analizado su influencia en el surgimiento de nuevas empresas (Beesley y Hamilton, 1984), se han trazado los coordenadas del debate de que han sido objeto (Acs y Audretsch, 1993), se han impulsado estudios institucionales que deriven en recomendaciones y políticas públicas (OCDE, 2010), se han investigado la influencia de factores intangibles, como la confianza, en sus procesos de innovación (Valenzuela, 2012) y se exponen los múltiples problemas que enfrentan (Escalera, 2007).

Conviene detenerse un instante en esta última línea ya que los problemas que esas empresas presentan suelen ser un freno para su desarrollo (es decir, para el incremento de su productividad, la mejora de la competitividad y de sus procesos de innovación) y las vuelve vulnerables a los cambios del medio ambiente económico y a la inestabilidad macroeconómica. Las Pymes enfrentan problemas de falta de crédito, de escasa información sobre fuentes de financiamiento, procesos administrativos rudimentarios, reducido margen de ganancia, uso de tecnologías obsoletas en la producción y deficientes controles de calidad. Además de eso, las pequeñas empresas se desenvuelven en medio de una muy pobre infraestructura productiva que padece el país.

\section{a) Innovación y vínculo con las CGV}

Conforme Sonora se ha convertido en asiento de empresas multinacionales en varias ramas de actividad (automotriz, aeroespacial, industria médica, etc.) la demanda de bienes

\footnotetext{
1 "El importante papel de las pequeñas empresas independientes, se afirma a menudo, es tener la función de semillero de nuevas empresas capaces de desafiar a las empresas establecidas.” (Beesley y Hamilton, 1984).

${ }^{2}$ Menkveld y Thurik (1999), aplicando un modelo que mide la relación entre tamaño de empresa y crecimiento, encontraron que una industria con baja presencia de grandes firmas (en comparación con las mismas industrias de otros países) se ha comportado mejor en términos de crecimiento del producto. En ese sentido, no reestructurar la industria (fomentando la competencia y la eliminación de monopolios), tiene un costo en términos de crecimiento.

${ }^{3}$ El libro clásico de Ronal Coase no trata explícitamente de pequeñas empresas, pero es evidente que no trata tampoco de grandes corporaciones. Es muy probable que el modelo que el autor tenía en mente haya sido la mediana empresa industrial.
} 
y servicios locales ha venido a constituir un mercado donde la competencia por los contratos se ha vuelto encarnizada. Para las Pymes sonorenses, convertirse en proveedor de una empresa transnacional no es tarea fácil; los requerimientos tecnológicos, organizacionales o de capacidades de producción e innovación que las grandes firmas exigen, además de que los convenios de colaboración regularmente presentan asimetrías de poder a favor de las ETN, que pueden ocasionar serios problemas para cumplir los compromisos adquiridos por parte de las empresas locales (Arias y Solari 2008; Bueno, 2012).

Como se puede suponer, hay ramas de actividad que vuelven a las empresas más proclives a participar en las redes de proveeduría. Es el caso de las metalmecánicas y de tecnologías de la información. El tipo de bienes y servicios que producen las convierten en actores del tipo de desarrollo local impulsado por las ETN (Contreras, 2005). Sin embargo, solamente una minoría de empresas (37.9\%) han logrado incorporarse a las cadenas de valor, siendo las MM las que proporcionalmente establecen con mayor frecuencia una relación de ese tipo al compararlas con las TI (véase tabla 1).

Tabla 1. Pymes por actividad de la empresa, según clientes transnacionales

\begin{tabular}{|c|c|c|c|}
\hline \multirow{2}{*}{$\begin{array}{c}\text { Clientes } \\
\text { Transnacionales }\end{array}$} & \multicolumn{2}{|c|}{$\begin{array}{l}\text { Actividad de la } \\
\text { empresa }\end{array}$} & \multirow[t]{2}{*}{ Total } \\
\hline & TI & MM & \\
\hline No & 75.0 & 50.0 & 60.3 \\
\hline Sí & 25.0 & 50.0 & 39.7 \\
\hline Total & 100.0 & 100.0 & 100.0 \\
\hline
\end{tabular}

Fuente: Transferencia de Conocimiento y Aprendizaje Tecnológico de las Pequeñas y Medianas Empresas (Pymes) locales en el entorno de las Redes Globales de Producción. UNISON, proyecto PRODEP No. 151329.

La explicación de esa diferencia se debe a que las ETN usan principalmente software proporcionado por sus matrices o de proveedores también internacionales, dejando a las empresas locales las actividades colaterales como mantenimiento y en algunos casos a instrumentar la adaptación de los programas a las condiciones locales.

En el caso de las MM, en cambio tienen otro fenómeno de contexto; las ETN encuentran preferible, en términos de costos, sustituir localmente las piezas metálicas desgastadas o la construcción de estructuras metálicas cuya transportación sería más costosa.

A pesar de que la mayoría de las empresas no tiene una relación con las ETN, han sido abundantes los estudios que analizan esa intersección entre las pequeñas empresas y las cadenas globales de valor (Contreras, Carrillo y Olea, 2012; Jurowetzki, Lundvall y Lema, 2015; Navarrete, Olea y Taddei, 2015; Olea, 2014). En particular, los aspectos que aquí interesa destacar en esa intersección son los referentes a los procesos de aprendizaje y la innovación tecnológica.

Sin embargo, lo que hace interesante el estudio de esos tópicos es que los impactos de las redes globales de producción son tanto directos como indirectos. La restructuración económica a partir de los años ochenta, que implicó que Sonora entrara en el nuevo modelo como eslabón manufacturero en las cadenas globales de producción, modificó la estructura productiva, dándole 
una orientación más clara hacia el exterior y amplió el campo de acción para una nueva generación de empresarios que se acomodaron a las nuevas condiciones económicas y se convirtieron en protagonistas en el desarrollo económico local (Bracamonte y Contreras, 2008).

Lo que la apertura de los mercados trajo es una aceleración de las tendencias innovadoras de las empresas locales, superando la vieja creencia de que las innovaciones se producían en los países industrializados, dejando a demás países la imitación y la adaptación de los nuevos desarrollos. En ese sentido, las Pymes locales que ingresan a las redes de proveeduría de las transnacionales absorben los conocimientos difundidos por éstas e incrementan sus capacidades generando innovaciones de producto, proceso, organizacionales y comercialización.

Debido quizá a los efectos indirectos de las redes globales de valor, una alta proporción de Pymes locales manifiestan haber implementado innovaciones de producto (76.5\%), de proceso $(69.1 \%)$, organizacionales $(48.5 \%)$ y de comercialización $(48.5 \%)$. Además, las pequeñas empresas proveedoras de las ETN reportan un mayor número de innovaciones y de tipos de innovación que las no proveedoras (tabla 2).

Un aspecto que parece lógico (y en la línea de las hipótesis planteadas) es que las empresas ligadas a las ETN son más innovadoras que las que no tienen relación de proveeduría, debido a que las empresas transnacionales exigen a las empresas locales altos estándares de calidad y operación que las obliga a un constante aprendizaje que se traduce en innovaciones.

Tabla 2. Pymes innovadoras por actividad y proveeduría hacia empresas transnacionales

\begin{tabular}{|c|c|c|c|c|c|c|c|c|c|}
\hline \multirow{4}{*}{$\begin{array}{l}\text { Es proveedor } \\
\text { de una ETN }\end{array}$} & \multicolumn{9}{|c|}{ Actividad de la empresa } \\
\hline & \multicolumn{3}{|c|}{ TI } & \multicolumn{3}{|c|}{ MM } & \multicolumn{3}{|c|}{ Total } \\
\hline & \multicolumn{9}{|c|}{ Realizó innovaciones de producto o proceso } \\
\hline & No & $\mathrm{Si}$ & Total & No & $\mathrm{Si}$ & Total & No & $\mathrm{Si}$ & Total \\
\hline No & 33.3 & 66.7 & 100.0 & 35.0 & 65.0 & 100.0 & 34.1 & 65.9 & 100.0 \\
\hline Sí & 0.0 & 100.0 & 100.0 & 0.0 & 100.0 & 100.0 & 0.0 & 100.0 & 100.0 \\
\hline Total & 25.0 & 75.0 & 100.0 & 17.5 & 82.5 & 100.0 & 20.6 & 79.4 & 100.0 \\
\hline \multicolumn{10}{|c|}{ Realizó innovaciones organizacionales o de comercialización } \\
\hline No & 52.4 & 47.6 & 100.0 & 55.0 & 45.0 & 100.0 & 53.7 & 46.3 & 100.0 \\
\hline Sí & 0.0 & 100.0 & 100.0 & 25.0 & 75.0 & 100.0 & 18.5 & 81.5 & 100.0 \\
\hline Total & 39.3 & 60.7 & 100.0 & 40.0 & 60.0 & 100.0 & 39.7 & 60.3 & 100.0 \\
\hline \multicolumn{10}{|c|}{ Realizó algún tipo de innovación } \\
\hline No & 33.3 & 66.7 & 100.0 & 35.0 & 65.0 & 100.0 & 34.1 & 65.9 & 100.0 \\
\hline Sí & 0.0 & 100.0 & 100.0 & 0.0 & 100.0 & 100.0 & 0.0 & 100.0 & 100.0 \\
\hline Total & 25.0 & 75.0 & 100.0 & 17.5 & 82.5 & 100.0 & 20.6 & 79.4 & 100.0 \\
\hline
\end{tabular}

Fuente: Transferencia de Conocimiento y Aprendizaje Tecnológico de las Pequeñas y Medianas Empresas (Pymes) locales en el entorno de las Redes Globales de Producción. UNISON, proyecto PRODEP No. 151329.

Los tipos de innovación considerados en la tabla 2 se pueden clasificar, a su vez, en las siguientes categorías: 1) incremental, que se refiere a la creación de valor añadido sobre un producto ya existente agregándole cierta mejora, y 2) radical, que se conoce como el cambio o introducción de un nuevo producto, servicio o proceso que no se conocía (OCDE, 2005). 


\section{Pequeñas empresas MM y de TI en Sonora ${ }^{4}$}

Según datos del Censo Económico de 2014, en Sonora hay 117,289 unidades económicas que dan empleo a 827,542 personas y las pequeñas empresas (hasta 100 trabajadores) representan 99.8 del total y demandan 72.8 por ciento del empleo. Las proporciones de esos números son en Sonora muy similares a los nacionales, según la misma fuente.

De acuerdo con Contreras (2005), el sector metalmecánico (que se agrupa en el 1.5\% de las unidades económicas y emplea al $2.01 \%$ del personal ocupado) está formado por un grupo de empresas que producen piezas y estructuras metálicas hasta la fabricación de maquinaria y equipo que involucran tecnología a la que podemos llamar "sofisticada" (herramientas de precisión, tornos digitales, robótica, etc.).

El 56 por ciento de las MM se dedican a servicios de mantenimiento industrial y de equipo técnico. Entre las MM, la actividad principal es la producción de piezas metálicas para la industria.

Las empresas de TI se ubican grosso modo en los servicios profesionales, científicos y técnicos, aunque las empresas desarrolladoras de software y de sistemas de cómputo (más en relación con los objetivos de esta investigación), son solo 72 empresas que contratan 962 empleados. Este es un sector más especializado y que "presenta un perfil tecnológico y empresarial más alto y una estrategia competitiva más enfocada hacia la calidad" (Contreras, 2005).

La industria del software ha sufrido un cambio radical en los últimos veinte años debido a dos procesos: primero, la generalización del uso de las tecnologías de la información y la comunicación y, segundo, la continua caída del precio de la parte tangible de esa tecnología (el hardware) y el incremento en el precio del software debido a su creciente sofisticación.

Además, el nivel de conocimientos requeridos por el personal de estas empresas es tan especializado que "son las que mayores dificultades reportan para conseguir los empleados que necesitan" (Contreras y Olea, 2006).

El promedio de edad de las plantas MM es de 17 años con una desviación estándar de 13.7. La MM más antigua data de hace 64 años. Esta información es importante para conocer el arraigo de la industria en el estado, pero para los efectos de esta investigación, un indicador más importante es la antigüedad promedio de la maquinaria y equipo. Según la información de campo recabada, el promedio de antigüedad en ambos giros de actividad (MM y de TI), es de aproximadamente 5 años. El mínimo de antigüedad es de cero años y el máximo de 20 . Sin embargo, consistente con el dato de la antigüedad de las empresas, en el sector de TI la edad de la maquinaria y equipo va de 0 a 8 años con una media de 2.29 años, mientras que en las de $\mathrm{MM}$ el rango va de 0 a 20 años con una media de 7.7, lo que se relaciona con un ciclo más breve de vida útil de los equipos de tecnologías de la información y con la menor antigüedad de las empresas: 17 años con una desviación estándar de 6.5, fundándose la primera empresa de este tipo hace unos 30 años.

\footnotetext{
${ }^{4}$ La información para este apartado proviene principalmente de la tesis de doctorado de Alejandro Valenzuela (2012) realizada en el marco del Proyecto No. 133596 del Consejo Nacional de Ciencia y Tecnología (CONACYT): Redes globales de producción y aprendizaje local: derrama tecnológica de las transnacionales y capacidad de absorción en PYMES de base tecnológica en el Noroeste de México, conducido conjuntamente por investigadores de El Colegio de la Frontera Norte y El Colegio de Sonora.
} 
A pesar de que el 86.5 por ciento de las empresas asegura haber realizado innovaciones, su propia calificación de la tecnología que usan es medianamente conservadora ya que alrededor de la mitad de las empresas aseguran que su tecnología está en el promedio y una tercera parte la ubica en la frontera tecnológica ${ }^{5}$. Debe decirse, sin embargo, que la proporción que está en la frontera tecnológica es mucho mayor en las de TI que en las MM, lo que se explica por el hecho de que en la industria metálica no es estrictamente necesario contar con la tecnología de última generación para permanecer en el mercado, mientras que la obsolescencia es una característica creciente en los equipos de cómputo y software.

Otro factor relevante para el estudio de la innovación en este tipo de empresas es el análisis del capital humano ${ }^{6}$. Al respecto, se consideran dos variables. Por un lado, la escolaridad del personal y, por otro, la formación profesional y la experiencia del empresario.

A pesar de que se trata de empresas en promedio muy pequeñas (19 trabajadores en las MM y 13 en las de TI), el nivel de escolaridad es relativamente alto. Los porcentajes de trabajadores con al menos licenciatura son de 52 en las de TI y de 15 en las MM. Para dimensionar esa información, veamos que la parte de la PEA que tiene ese nivel de escolaridad es de tan sólo $13 \%$ a nivel nacional y de $14 \%$ en Sonora. Lo que se observa es que, sobre todo las empresas de TI se despegan considerablemente del promedio.

Respecto a los empresarios de los sectores analizados, la inmensa mayoría de ellos (68\% en MM y $93 \%$ de los de TI) tienen estudios de licenciatura o superiores. (A nivel estatal, solamente una tercera parte tiene ese nivel de escolaridad, de acuerdo con Covarrubias, 2000).

La escolaridad en este sector no se debe al "abolengo" de los empresarios, que no existe, sino a la necesidad de forjarse un lugar entre los empresarios locales ya que el 80 por ciento de ellos han sido empleados calificados en otra compañía antes de ser empresarios. Esto se puede interpretar como una toma de experiencia en actividades relacionadas con su función actual y que les ha ayudado a fundar una empresa con cierto nivel de éxito. Apunta hacia esta hipótesis el hecho de que la mayoría de ellos trabajó como responsables de producción, mantenimiento y de proyectos. Digamos que la experiencia previa fue un proceso de adquisición de conocimientos y un aprendizaje útil para un negocio con ciertas ventajas (véase Tabla 3 ).

\footnotetext{
${ }^{5}$ La teoría económica supone que las empresas operan con la tecnología estándar mostrada por la función de producción. Todas las técnicas productivas sub-estándar estarían, en un modelo de equilibrio general, por debajo de la frontera de posibilidades de producción y estarían usando combinaciones de insumos no óptimas en el sentido de Pareto. Ese sólo hecho sería suficiente para avanzar hacia el equilibrio eficiente o salir del mercado (Gravelle y Rees, 1996). Sin embargo, esta hipótesis neoclásica no se verifica en el mercado que estamos analizando porque las empresas con tecnologías por debajo de la frontera tecnológica siguen operando debido, quizá, al sacrificio de planes de ganancia a corto plazo en aras de la competitividad a través de la innovación a lo que se hace referencia en el primer apartado de este capítulo. Se debe decir que la clasificación no es producto de una tipología tecnológica, sino del conocimiento que tiene el empresario sobre el entorno de su giro de actividad y de la comparación de su equipo con el existente en el medio y en los países de mayor desarrollo en el área.

${ }^{6}$ Dice Theodore W. Schultz en su influyente artículo de 1961 sobre capital humano: "Ha sido ampliamente observado que los incrementos en el producto nacional han sido grandes comparados con los incrementos en tierra, horas de trabajo y capital físico reproducible. La inversión en capital humano ha sido la principal explicación de esa diferencia". Por su parte, Gary Backer, en su nota sobre capital humano para The Concise Encyclopedia or Economic, que el capital no sólo es esa forma tangible que se conoce: "la escolaridad, los cursos de entrenamiento en computadoras, los gastos médicos, las enseñanzas sobre puntualidad y honestidad son también capital. Eso es porque esos factores incrementan los ingresos... Se les llama capital humano porque la gente no puede ser separada de sus conocimientos, habilidades, salud o valores en el sentido en que puede ser separada de sus activos físicos y financieros."
} 
Tabla3. Pymes por actividad de la empresa y proveeduría de empresa transnacional, según experiencia laboral del dueño

\begin{tabular}{|c|c|c|c|c|c|c|c|c|c|}
\hline \multirow{4}{*}{$\begin{array}{l}\text { El dueño trabajó en } \\
\text { alguna otra empresa o } \\
\text { institución }\end{array}$} & \multicolumn{9}{|c|}{ Actividad de la empresa } \\
\hline & $\mathrm{TI}$ & & & MM & & & Total & & \\
\hline & \multicolumn{9}{|c|}{ Es proveedor de una empresa transnacional } \\
\hline & No & Sí & Total & No & Sí & Total & No & Sí & Total \\
\hline Sí & 90.5 & 85.7 & 89.3 & 75.0 & 90.0 & 82.5 & 82.9 & 88.9 & 85.3 \\
\hline No & 9.5 & 14.3 & 10.7 & 25.0 & 10.0 & 17.5 & 17.1 & 11.1 & 14.7 \\
\hline Total & 100.0 & 100.0 & 100.0 & 100.0 & 100.0 & 100.0 & 100.0 & 100.0 & 100.0 \\
\hline
\end{tabular}

Fuente: Transferencia de Conocimiento y Aprendizaje Tecnológico de las Pequeñas y Medianas Empresas (Pymes) locales en el entorno de las Redes Globales de Producción. UNISON, proyecto PRODEP No. 151329.

\section{Metodología}

Los fundamentos teóricos de esta investigación son, primero, los sistemas de innovación y la discusión correspondiente sobre el papel del conocimiento y del aprendizaje en el desarrollo local (Lundval, 2007); segundo, la propuesta de cadenas globales de valor, que destaca la articulación productiva global de las ETN y las empresas locales (Gereffi, Humphrey y Sturgeon 2005; Pietrobelli y Rabelloti, 2009) y que ofrece un marco analítico de los mecanismos de aprendizaje tecnológico que permiten a las empresas locales participar en las redes de abastecimiento de las transnacionales (Bracamonte y Contreras, 2008), y tercero, la discusión sobre el aprendizaje tecnológico que pasa de explícito a implícito en función de la distancia cognitiva (Nooteboom, 2000) y de las capacidades potenciales y reales de aprendizaje (Zahara y George, 2002). Debe decirse, respecto a esto último, que el aprendizaje y las capacidades de absorción no "detonan" de manera espontánea, sino por la presencia de un conjunto de factores (como la competitividad) que trae la presencia de algún agente, generalmente externo, que induzca a los empresarios a pensar en la necesidad de nuevos conocimientos que le permitan introducir cambios para mejorar sus procesos, aumentar su capacidad de producción, reducir costos, mejorar su tecnología o innovar (Arechavala, 1998; González-Campo y Hurtado, 2014; Olea, 2014) para convertirse en proveedor de las ETN y mejorar así sus probabilidades de permanencia en el mercado (Hauschild, Licht y Stein, 2001; Orr, 2008).

Se debe tener en cuenta que se trata de un estudio transversal y, por tanto, no estamos en condiciones de analizar el comportamiento histórico de la relación entre las Pymes MM y de TI, por un lado, y las ETN por otro, en el asunto de la innovación. En este sentido deberemos atenernos a los hallazgos de investigaciones que se han hecho al respecto en el medio local de la industria sonorense (Bracamonte y Contreras, 2008; Contreras, 2005 y 2008; Contreras y Olea, 2006) y a los resultados de los cuestionarios aplicados donde se reporta la antigüedad de la empresa, de la maquinaria y la trayectoria laboral del empresario.

\section{a) La muestra y los indicadores}

Los resultados de la investigación se basan en las pruebas de un modelo de regresión logística en el que se ha usado información proveniente de la aplicación de un cuestionario estructurado a una muestra de empresas de las ramas MM y TI asentadas en cuatro municipios 
del estado de Sonora ${ }^{7}$. Se trata de una investigación de tipo transversal y no experimental en la que se busca establecer la relación estructural entre la variable dependiente "probabilidad de participación de la empresa en las redes de proveeduría de las empresas transnacionales" y los componentes de los diversos tipos de innovación que generan las empresas.

La selección de las empresas se debe a las razones expuestas en el apartado b del punto 3 . El listado completo de empresas de ambas ramas de actividad proviene de las bases de datos de la Cámara Nacional de la Industria Electrónica, de Telecomunicaciones y Tecnologías de la Información, de los listados de empresas de los parques industriales de Sonora de la Secretaría de Economía, de los registros de la Cámara Nacional de la Industria de la Transformación y de la página web de la Sección Amarilla. La selección se hizo en los municipios de Hermosillo, Guaymas y Empalme por las razones expuestas en la sección c del punto 3) después de la depuración de los listados para eliminar empresas que no cumplieran los requisitos de tamaño (pequeñas empresas), que produjeran piezas metálicas, máquinas y herramientas para la industria (lo que excluye a los talleres de herrería), que desarrollaran sistemas de cómputo y software (lo que excluyó a los cafés internet, a las radiodifusoras y a los talleres de vena y reparación de equipo). Al final, de 410 empresas, fueron seleccionadas 68 empresas ( 28 de TI y $40 \mathrm{MM}$ ) de acuerdo con un muestreo aleatorio estratificado.

Elegida la muestra, la aplicación del modelo tiene sentido solamente a partir de indicadores claramente definidos, que sean el resumen de conceptos multidimensionales, y de escalas de medición que reflejen con claridad las mediciones nominales, ordinales o por intervalo que se usen en el análisis (Schuschny y Soto, 2009; Venkatraman y Grant, 1986).

Existen diferentes técnicas para la construcción de indicadores. Aquí se han construido factores por medio de la suma simple de variables previamente estandarizadas para que sus escalas de medición sean comparables y agregables.

El primer paso consistió en seleccionar y estandarizar las variables para conformar los factores de acuerdo con cada tipo de innovación: de producto, proceso, organizacionales y comercialización. Con estos factores se construyeron dos índices: uno para las innovaciones agrupadas en dos grandes categorías, una de producto y de proceso y otra para las innovaciones administrativas y de comercialización.

\section{b) Técnicas y modelos estadísticos}

Dado que esta es una investigación de tipo cuantitativo, los objetivos del uso de los modelos estadísticos son: 1) entender las características de las variables y 2) hacer pronósticos sobre las relaciones de dependencia entre ellas validando los resultados (Hair, Bush y Ortinau, 2010).

Una vía apropiada para verificar la hipótesis de que a mayor innovación de diferentes tipos, mayor es la probabilidad de que las pequeñas empresas tengan una relación de proveeduría con las empresas transnacionales y las cadenas globales de valor a las que pertenecen, es la regresión lineal que establece una relación de causalidad entre variables. En particular, y dado que la variable dependiente es una variable dicotómica (sí o no participa la empresa en las redes de proveeduría de las ETN), lo que al final determina la probabilidad de que eso suceda en función de otra variable, en este caso la innovación, el modelo apropiado es el de regresión logística.

\footnotetext{
${ }^{7}$ El cuestionario (titulado "Transferencia de Conocimiento y Aprendizaje Tecnológico de las Pequeñas y Medianas Empresas locales en el entorno de las Redes Globales de Producción") contiene 40 preguntas en tres secciones: I. Datos generales, II. Capacidades de Innovación y III. Relación de las innovaciones con las empresas transnacionales. Antes del levantamiento formal de la información se realizaron pruebas piloto para validar el instrumento.
} 
Este modelo no requiere de los supuestos de normalidad, es de probabilidad lineal, permite el uso de variables independientes continuas y categóricas, tiene capacidad de incorporar efectos no lineales y es útil para realizar predicciones y diagnósticos (Alderete, 2006; Calderón y De los Godos, 2009; García, Alvarado y Jiménez, 2000; Hair, Anderson, Taham y Black, 1999; Llaugel y Fernández, 2011; Nava y Pradad, 2008).

Si Y es la variable dependiente y si $\mathrm{X}\left(\mathrm{X}_{1}, \mathrm{X}_{2}, \mathrm{X}_{3}, \ldots, \mathrm{X}_{\mathrm{k}}\right.$, ) son las variables explicativas, el modelo se puede platear asís:

$$
\operatorname{Pr}(Y=1)=\frac{1}{1+\theta^{-x}}
$$

Donde $\quad X=\beta_{0}+\beta_{1} X_{1}+\beta_{2} X_{2}+\cdots+\beta_{k} X_{k}$

No está por demás mencionar que el valor de los coeficientes $\beta$ i (suponiendo cubiertas todas las pruebas estadísticas que validan el modelo) tiene un peso específico en la interpretación de los resultados: si es positivo indicará que la probabilidad de ser proveedor de una ETN aumenta al incrementarse las capacidades de innovación, y viceversa.

\section{Análisis y discusión de resultados}

En este apartado se presentan de manera desagregada los componentes y estructura del modelo de análisis propuesto y sus resultados para el contraste y validación de las hipótesis de la investigación. Los tabulados, cálculos y estimaciones que se muestran a lo largo de este capítulo, tienen como fuente la base de datos del cuestionario propuesto.

\section{Construcción del modelo}

Dado que el objetivo de la investigación es analizar la probabilidad de ocurrencia de que una empresa local de las ramas MM y de TI tenga como cliente a una empresa transnacional, probabilidad que está en función del número de tipos de innovaciones que desarrollan y aplican, el mejor modelo que realiza ese pronóstico es uno de regresión logística (Gail, 2010).

La variable dependiente (si la Pyme MM o de TI es o no proveedora de una empresa transnacional) es dicotómica en la que si el evento ocurre, $\mathrm{Y}=1$; de lo contrario, $\mathrm{Y}=0$.

Las variables independientes o predictoras están compuestas por los índices de innovación construidos con los cuatro tipos reconocidos: de producto, procesos, organizacionales y comercialización.

Como se sabe, este tipo de modelos de regresión tiene una variable confundente que en el caso presente es la actividad de la empresa (cuya codificación es 1 para MM y 0 para TI). Es una variable independiente de efecto externo a la relación principal (tipos de innovación® ser

\footnotetext{
${ }^{8}$ Para una exposición sucinta del modelo de regresión logística, vea el capítulo 15 del libro de Econometría de Damodar N. Gujarati y Dawn C. Porter (2009). Ver también Gail, Krickeberg, Samet y Tsiatis, (2010).
} 
proveedor de empresa transnacional) porque las actividades de producción y administración de las empresas analizadas son distintas.

La estimación del modelo es la siguiente:

Tabla 4. Estadísticos de resultados de la regresión logística ${ }^{\mathbf{a}, \mathbf{b}}$

\begin{tabular}{cccccccccc}
\hline & & & & & & & & \multicolumn{2}{c}{ I.C. 95\% para EXP(B) } \\
\cline { 7 - 10 } & & E.T. & Wald & gl & Sig. & Exp(B) & Inferior & Superior \\
\hline \multirow{3}{*}{ Paso 1a } & IOC & 4.733 & 2.046 & 5.353 & 1 & .021 & 113.638 & 2.062 & 6263.386 \\
& IPP & 4.825 & 1.567 & 9.479 & 1 & .002 & 124.592 & 5.774 & 2688.494 \\
& Actividad(1) & -2.101 & .892 & 5.545 & 1 & .019 & .122 & .021 & .703 \\
& Constante & -2.663 & .782 & 11.610 & 1 & .001 & .070 & & \\
\hline
\end{tabular}

a El punto de corte es 0,500
b El signo positivo en el exponente indica un aumento en la probabilidad de ocurrencia del evento; un signo negativo la reduce y un coeficiente cercano a cero resulta en un valor próximo a la unidad que prácticamente no incide en la probabilidad de ocurrencia del evento (Ibarra y Michalus, 2010)

El modelo mostrado en la Ecuación 1, sustituyendo los resultados mostrados en la tabla 4, es siguiente:

$$
\operatorname{Pr}(Y=1)=\frac{1}{1+e^{-x}}
$$

\section{Donde}

$\mathrm{P}(\mathrm{Y}=1)=$ Probabilidad de la pyme sea proveedor de una empresa transnacional

$$
X=-2.663+4.733 I O C+4.825 I P P-2.101 A
$$

IOC $=$ Tipos de innovaciones organizacionales y de comercialización

IPP = Tipos de innovaciones de producto y proceso

A= Actividad principal de la PYME (MM o TI)

Los datos presentados podemos interpretarlos de la siguiente manera. El valor de la constante $\left(\beta_{0}\right)$ no tiene interpretación propia, se considera un valor de ajuste que debe ser incluido en el modelo. Los valores de la constantes $\beta_{1}=4.773$ y $\beta_{2}=4.825$ ambos positivos nos indican que la probabilidad de ser proveedor de una ETN, aumenta al incrementarse los valores de las variables; en este caso, referida a los tipos de innovaciones de producción (IPP = producto y proceso) y administración (IOC $=$ organizacionales y de comercialización) que realizan las Pymes locales. El valor de la constante $\beta_{3}$ está referido a la actividad principal de las Pymes, que por ser una variable dicotómica y de valor negativo $(-2.101)$, indica que la probabilidad de ser proveedor de una ETN se reduce en función del giro de actividad. Así, una empresa de MM (a la que se le asignó el valor de 1), tienen menores probabilidades de ser proveedora que una TI, a la que se le asignó el código 0 ). 
Además, los estimadores de los parámetros de las tres variables independientes son estadísticamente significativos ya que el nivel de significancia asociada al índice de Wald es, en todos los casos, menor que 0.03. Los signos de los coeficientes permiten inferir que las variables IOC e IPP afectan positivamente la probabilidad de que las PYMES sean proveedoras de una ETN.

La ecuación 2 nos permite predecir la probabilidad de que una Pyme sea proveedora de una ETN a partir de los tipos de innovaciones de producto, proceso, organizacionales y de comercialización que ha realizado y su actividad económica. Esta regresión logística proporciona el cálculo de probabilidades de ocurrencia de que una Pyme sea proveedora de una ETN entre todas las Pymes de la muestra. Si el ajuste es bueno, se espera que la presencia de tipos de innovación implantadas se asocie con valores altos de probabilidad de que la empresa sea proveedora de ETN.

En la tabla 5 se presentan probabilidades de las Pymes de TI y MM sean proveedoras de una empresa transnacional en función de los tipos de innovación implementadas. Con esos resultados podemos inferir que la probabilidad de que una pequeña empresa sea proveedora de una empresa transnacional, estará en función del número de tipos de innovaciones que haya implementado y del giro de actividad económica.

Tabla 5. Probabilidad de la pyme de ser proveedor de una ETN según sus tipos de innovación implementados

\begin{tabular}{llcc}
\hline \multicolumn{2}{c}{ Innovación } & \multicolumn{2}{c}{ Actividad de la empresa } \\
\hline OC & PP & TI & MM \\
0.0 & 0.0 & 0.065 & 0.012 \\
0.1 & 0.1 & 0.153 & 0.028 \\
0.2 & 0.2 & 0.320 & 0.093 \\
0.3 & 0.3 & 0.551 & 0.335 \\
0.4 & 0.4 & 0.761 & 0.690 \\
0.5 & 0.5 & 0.892 & 0.871 \\
0.6 & 0.6 & 0.956 & 0.936 \\
0.7 & 0.7 & 0.982 & 0.964 \\
0.8 & 0.8 & 0.993 & 0.978 \\
0.9 & 0.9 & 0.997 & 0.987 \\
1.0 & 1.0 & 0.999 & 0.992 \\
\hline
\end{tabular}

Fuente: Transferencia de Conocimiento y Aprendizaje Tecnológico de las Pequeñas y Medianas Empresas (Pymes) locales en el entorno de las Redes Globales de Producción. UNISON, proyecto PRODEP No. 151329.

Las capacidades de innovación que desarrollan las Pymes locales pueden ser un factor fundamental para que estén en condiciones de cumplir con los parámetros que les imponen las empresas transnacionales para ser aceptadas como sus proveedores. Sin embargo, no siempre esas exigencias son los únicos factores que juegan a favor (o en contra) de la participación de las Pymes. También están los factores contingentes; en entrevistas cara-a-cara con pequeños empresarios locales queda de manifiesto que la presencia de un problema inesperado (fallas mecánicas, atascos en la línea de producción, etc.) obliga a las grandes empresas a buscar 
soluciones inmediatas con empresas locales, lo que las coloca en la ruta de la proveeduría y las alienta a buscar implantar innovaciones en su propio desempeño.

\section{Conclusiones}

De acuerdo con los estudios citados en este trabajo, son muchas las ventajas de que una pequeña empresa local ingrese en las redes de proveeduría de las firmas multinacionales. La probabilidad de que lo logre, como se demuestra, aumenta si la Pyme local genera innovaciones de varios tipos porque eso incrementa su competitividad y la pone en condiciones de cumplir con los estándares de calidad y tiempo que esas empresas exigen.

Sin embargo, tener éxito en la búsqueda de la mejora de la capacidad de innovación de una pyme resulta ser un proceso complicado, por varios motivos. En primer lugar, para que una Pyme pueda desarrollar actividades innovadoras requiere superar una serie de obstáculos internos tales como deficientes sistemas de producción, tecnología obsoleta, pobre capacitación y pobre nivel educativo de trabajadores y dueños, entre otros. Esas deficiencias impiden o retrasan los procesos de adquisición del conocimiento que puede provenir del entorno o que es trasmitido por las firmas líderes de las cadenas de valor. En segundo lugar, es común que las empresas que emprenden esfuerzos innovadores se concentren en lograr innovaciones de producto o de proceso. El objetivo de esta investigación fue demostrar que para que una pyme local sea considerada como proveedora de una ETN requiere desarrollar e implementar varios tipos de innovación de producto, proceso, comercialización y organizacionales.

Los resultados de este estudio demuestran que las pymes sonorenses de las ramas MM y de TI que exhibieron actividades de innovación en el espectro más amplio, incrementaron su probabilidad de ser aceptadas en la red de proveeduría de las grandes trasnacionales establecidas en la región. Adicionalmente, se observó que las Pymes de TI poseen mayores probabilidades de incorporarse a las cadenas globales de valor.

Coincidimos en la afirmación de Lall (1992), cuando dice que, para la mayoría de las Pymes, hacer negocios fuera de los mercados que constituyen su entorno geográfico inmediato es un reto mayor, se considera que la internacionalización de la empresa es un proceso evolutivo, regularmente lento y crece en medida que se desarrollan sus capacidades. Si una empresa busca vender sus productos o servicios fuera de su ámbito local, debe diseñar una estrategia de comercialización en mercados nacionales o internacionales, porque el nivel de competitividad se ha visto afectado por la globalización.

Encontramos que son escasas las Pymes que implementan innovaciones de comercialización para incursionar en el mercado global; sus preocupaciones están más encaminadas a realizar mejoras en sus productos, procesos o servicios que les permitan mantenerse o sobrevivir en el mercado local. El proceso de incorporación a los mercados globales, es particularmente difícil para ellas por carecer de conocimientos y recursos que les permitan exportar sus productos o servicios fuera de su entorno; para este tipo de empresas, la internacionalización radica en poseer como clientes a empresas transnacionales que incorporen su producción a la cadena de valor o, en menor medida, que la gran empresa comercialice los productos, en ámbitos geográficos que serían de difícil acceso para ellas.

Las oportunidades de convertirse en proveedor de una ETN son escasas para las Pymes locales, sin embargo, se presentan oportunidades a partir de las propias estrategias de estas 
grandes empresas, por ejemplo, con la finalidad de minimizar costos o de resolver problemas inmediatos, buscan empresas locales que posean las capacidades necesarias para solventarlos.

Un importante hallazgo que muestran los resultados del modelo de regresión logística, es que las Pymes sonorenses de MM, proporcionalmente se vinculan más que las de TI, pero estas últimas tienen mayores probabilidades de ser elegidas como proveedoras de empresas transnacionales, fenómeno que puede ser explicado en función del tipo de innovaciones implementadas, las actividades propias de las empresas y de las necesidades de las ETN.

Las capacidades que las empresas transnacionales les solicitan a las Pymes locales, son una barrera poderosa para desistir en el intento de convertirse en proveedoras. Sin embargo, algunas veces se presentan problemas en las líneas de producción, relacionados con fallas mecánicas, que las obligan a buscar soluciones inmediatas con empresas locales de metalmecánica. Por otra parte, el contexto geográfico del estudio empírico donde se desarrolla esta investigación, tiene la característica de su cercanía con el mercado de Estados Unidos, país donde se encuentran la mayoría de las casas matrices de las transnacionales con representaciones asentadas en la región, situación que favorece a las empresas de MM en comparación a las de TI, porque las filiales de las grandes empresas se sujetan a los requerimientos de su matriz en cuanto a sistemas de información, donde su implementación y mantenimiento depende en mayor medida de las empresas vinculadas con la matriz, por lo que resulta difícil que una ETN asentada en la región, solicite los servicios de una empresa de TI local.

En resumen, el modelo de regresión logística, permite diferenciar tres condiciones básicas, 1) la probabilidad de que una empresa local sea elegida como proveedor de una transnacional, está directamente relacionada con los tipos de innovación que ésta ha implementado; 2) las Pymes de MM proporcionalmente se vinculan más que las de TI y, 3) las empresas de TI tienen mayores probabilidades que las de $\mathrm{MM}$, de ser elegidas como proveedoras de las grandes empresas.

Sin duda, la búsqueda del desarrollo de las pequeñas y medianas empresas nacionales debe ser un imperativo en la agenda de los tomadores de decisiones gubernamentales ya que es preciso impulsar políticas públicas que impulsen la mejora de las Pymes a través de una mayor vinculación con instituciones de educación superior y que fomenten el encadenamiento de la industria local con empresas trasnacionales.

\section{Referencias}

Acs, Z. J. \& Audretsch, D. B. editors. (1993). Small Firms and Entrepreneurship. En East-West perspective. Cambridge University Press.

Alderete, A. M. (2006). Fundamentos del Análisis de Regresión Logística en la Investigación Psicológica. Evaluar, $6,52-67$.

Arechavala, V. R. (1998). Formas de operación en pequeñas empresas del occidente de México: Los retos de la competitividad en mercados abiertos. Contaduría y Aministración, 198, 66-84.

Arias, T. D. \& Solari, V. A. (2008). Microempresas y Cadenas Transnacionales. El Caso de Walmart en Michoacán. Economía y Sociedad, 14 (21), 111-129.

Armenteros, A. MC., Medina, E. M., Ballesteros, M. L.L. \& Molinas, M. V. (2012). Las prácticas de gestión de la innovación en las micro, pequeñas y medianas empresas: resultados del estudio de campo en Piedras Negras Coahuila, México. Revista Internacional Administración y Finanzas, 5(4), 29-50. 
Berger, S. \& Piore, M. J. (1980). Dualism and Discontinuity in Industrial Societies. Cambridge University Press.

Beesley, M.E. \& Hamilton, R. T. (1984). Small firms' seedbed and the concept of turbulence. The Journal of Industrial Economics, 33(2). DOI en: https://doi.org/10.2307/2098510

Bracamonte S. Á. \& Contreras, O. (2008). Redes globales de producción y proveedores locales: los empresarios sonorenses frente a la expansión de la industria automotriz. Estudios Fronterizos, 9(18), 161-194.

Brok, W. A. \& Evans, D. E. (1989). Small Business Economics. Small Business Economics No. 1. Netherlands. DOI en: https://doi.org/10.1007/bf00389913

Bueno, C. C. (2012), La selección de los más aptos: evolución de las Pymes basadas en tecnología hacia la generación de soluciones productivas más complejas. En El Colegio de la Frontera Norte y Complejidad, Ciencia y Sociedad (Eds.), Dilemas de la innovación en México, dinámicas sectoriales, territoriales e institucionales (pp.185-224). México.

Calderón, S. J. P. \& De los Godos, U. L. A. (2009). Regresión logística aplicada a la epidemiología. Revista Salud, Sexualidad y Sociedad, 1(4), 1-9.

Censos Económicos 2014, INEGI, (2014). Censos Económicos 2014, Datos relevantes de los resultados definitivos [consultado 18 Ene 2016]. Disponible en:: http://www.inegi.org.mx/saladeprensa/boletines/2015/especiales/especiales2015_07_5.pdf

Coase, Ronald H. (1937). The nature of the firm. Econometrica, Vol. 4. Noviembre de 1937. DOI en: https://doi. org $/ 10.2307 / 2626876$

Contreras, O. F. (2005). Impacto de la ampliación de a Ford Motor Co. en Hermosillo, Sonora. Secretaría de Economía, El Colegio de Sonora, Fumec.

Contreras, O. F. (2008). Pequeñas empresas globales: un conglomerado automovilístico en México. Comercio Exterior, 58(8), 617-629.

Contreras, O. F. \& Olea, M. J. (2006). Estudio sobre el impacto de la ampliación de la Ford Motor Co. en Hermosillo, Sonora. Fundación México-Estados Unidos para la Ciencia, El Colegio de Sonora.

Contreras, O. F., Carrillo, J. \& Olea, M. J. (2012), Desprendimiento de las multinacionales, una vía para el aprendizaje y la innovación en empresas locales?. En El Colegio de la Frontera Norte y Complejidad, Ciencia y Sociedad (Eds.), Dilemas de la innovación en México, dinámicas sectoriales, territoriales e institucionales (pp.301-336).

Covarrubias, A. (2000). La viabilidad económica de Sonora. Ignacio Almada (eds). En Sonora 2000 a debate. El colegio de Sonora.

Escalera. Ch. M. E. (2007). Características organizacionales e individuales de los dueños o administradores de las PYMES. Decisiones financieras y la maximización del valor de la empresa. Tesis doctoral, Universidad Autónoma de San Luis Potosí. México.

Gail, K. M., Krickeberg, J.M. Samet, A. \& Tsiatis, W. W. (Eds.). (2010). Logistic Regression: A Self-Learning Text. Springer. DOI en: https://doi.org/10.1007/978-1-4419-1742-3

García, J. M. V., Alvarado, I. J. M. \& Jiménez, B. A. (2000). La predicción del rendimiento académico: regresión lineal versus regresión logística. Psicothema, 12(2), 248-525. [consultado 23 Ene 2016]. Disponible en: http://www. redalyc.org/articulo.oa?id=72797059

Gary S. Becker. (2008). Human Capital. The Concise Encyclopedia of Economics. Library of Economics and Liberty. DOI en: https://doi.org/10.1017/cbo9780511599040.013

Gereffi, Gary, John Humphrey \& Timothy Storgeon (2005). The governance of global value chains. Review of International Political Economy. DOI en: https://doi.org/10.1080/09692290500049805

González-Campo, C. H. \& Hurtado, A. A. (2014). Influencia de la capacidad de absorción sobre la innovación: un análisis empírico en las mipymes colombianas. Estudios Gerenciales, 30(2014), 277-286 DOI en: https://doi.org/10.1016/j.estger.2014.02.015

Gravelle, H. \& Rees, R. (1996). Microeconomics. Second Edition. Addison Wesley Longman Limited. London.

Guiarati, D. N. \& Porter, D. C. (2010). Econometría. Quinta Edición. Mc Graw-Hill.

Hair, J. F., Anderson, R. E., Tatham, R. L. \& Black, W. C. (1999). Análisis. México: Pearson Prentice Hall.

Hair, J. F., Bush, R. P. \& Ortinau, D. J. (2010). Investigación Secundaria: diseños, indagaciones y fuentes. Investigación de mercados (pp. 106-141). México: Mc Graw Hill.

Hauschild, H., Licht, T. \& Stein, W. (2001). Creating a Knowledge culture. McKinsey \& Company. 1, 74-81. 
Ibarra. M.C. \& Michalus, J. C. (2009). Análisis del rendimiento académico mediante un modelo logit. Ingeniería Industrial. 9(2), 47-56.

Jurowetzki, R., Lundvall, B. \& Lema, R. (2015). Combining the Global Value Chain and the Innovation System perspectives. Paper toe b presented at the DRUID Academy conference in Rebil, Aalborg, Denmark on January.

Lall, S. (1992), Technological Capabilities and Industrialization, World Development, 20(2) DOI en: https://doi.or$\mathrm{g} / 10.1016 / 0305-750 x(92) 90097-\mathrm{f}$

Llaugel, F. A. \& Fernández, A. M. (2011). Evaluación del uso de modelos de regresión logística para el diagnóstico de instituciones financieras. Ciencia y Sociedad, 36(4), 590-627 [consultado 20 Ene 2016]. Disponible en: http:// www.redalyc.org/articulo.oa?id=87022786002 DOI: https://doi.org/10.22206/cys.2011.v36i4.pp590-627

Lundvall, B. (2007). National innovation system: Analytical concept and development tool. Industry and Innovation, 14(1), 95-119. DOI en: https://doi.org/10.1080/13662710601130863

Matsuyama, K. (1995). Complementarities and Cumulative Processes in Models of Monopolistic Competition, Journal of Economic Literature, 33, 701-729.

Menkveld, B. \& A.R. Thurik (1999), Firm size and efficiency in innovation, Small Business Economics, 12(1): 97-101 DOI en: https://doi.org/10.1023/a:1008072817640

Nava, P. L. \& Pradad, S.S. (2008). Ajuste e interpretación de modelos de regresión logística con variables categóricas y continuas. Universidad Médica Bogotá, Colombia, 49(1), 46-57.

Navarrete, H. M.A., Olea, M. J. \& Taddei, B. J. (2015). Importancia de las políticas industriales para el desarrollo de la capacidad de innovación en PYMES, INVURNUS, 10(1), 27-32.

Nelson, R. \& Winter, S. G. (1982). An Evolutionary Theory of Economic Change. MA: Harvard University Press.

Nooteboom, B. (2000). Learning and Innovation in Organizations and Economies. Oxford University Press. NY.

OCDE (2005). Manual de OSLO tercera edición, Guía para la recogida e interpretación de datos sobre innovación. OCDE Eurostat.

OCDE (2010). SMEs, Entrepreneurship and Innovation. OCDE Studies on SMEs and Entrepreneurship.

Olea, M. J. (2014). Las capacidades de absorción del conocimiento como ventaja competitiva para la inserción a las cadenas globales de valor: las PYMES de tecnologías de información y metalmecánica en Sonora, México (Tesis Doctoral). Universidad Popular Autónoma del Estado de Puebla. Puebla, Puebla, México.

Orr, G. (2008). Seven ways China might surprise us in 2009. Mckinsey Quarterly, 1-3.

Pietrobelli, C. \& Rabellotti, R. (2009). Innovation systems and global value chains. WP SERIES, 3(9), 1-18.

Schultz, T. W. (1961). Investment in Human Capital. The American Economic Review, 51 (1), 15-32.

Storey D. J. (1994). Understanding the Small Business Sector. International Thomson Business Press. UK.

Schuschny, A. \& Soto, H. (2009). Guía metodológica diseño de indicadores compuestos de desarrollo sostenible. Comisión Económica Para América Latina y El Caribe. CEPAL [consultado 23 Ene 2016]. Disponible en: http:// repositorio.cepal.org/bitstream/handle/11362/3661/1/S2009230_es.pdf

Venkatraman, N. N. \& Grant, J. H. (1986). Construct Measurement in Organizational Strategy Research: A Critique and Proposal. Academy of Management Review, 11(1), 71-87. DOI en: https://doi.org/10.5465/amr.1986.4282628

Valenzuela, A. (2012). Confianza e innovación tecnológica de las pequeñas empresas metalmecánicas y de tecnologías de la información de Sonora (Tesis doctoral). El Colegio de Sonora.

Zahra, S. A. \& George, G. (2002). Absorptive capacity: A Review, reconceptualization, and extension. Academy of Management Review, 27(2), 185-203. DOI en: https://doi.org/10.5465/amr.2002.6587995 